\title{
Enhanced spin-orbit optical mirages from dual nanospheres
}

\author{
Jorge Olmos-Trigo, ${ }^{1}$ Cristina Sanz-Fernández, ${ }^{2}$ Aitzol García-Etxarri, ${ }^{1,2,3}$ Gabriel Molina-Terriza, ${ }^{1,2,3}$ \\ F. Sebastián Bergeret, ${ }^{1,2}$ and Juan José Sáenz ${ }^{1,3, *}$ \\ ${ }^{1}$ Donostia International Physics Center (DIPC), 20018 Donostia-San Sebastián, Spain \\ ${ }^{2}$ Centro de Física de Materiales (CFM-MPC), Centro Mixto CSIC-UPV/EHU, 20018 Donostia-San Sebastián, Spain \\ ${ }^{3}$ IKERBASQUE, Basque Foundation for Science, 48013 Bilbao, Spain
}

(Received 13 July 2018; published 29 January 2019)

\begin{abstract}
Spin-orbit interaction of light can lead to the so-called optical mirages, i.e., a perceived displacement in the position of a particle due to the spiraling structure of the scattered light. In electric dipoles, the maximum displacement is subwavelength and does not depend on the optical properties of the scatterer. Here we will show that the optical mirage in high refractive index dielectric nanoparticles depends strongly on the ratio between electric and magnetic dipolar responses. When the dual symmetry is satisfied (at the first Kerker condition), there is a considerable enhancement (far above the wavelength) of the spin-orbit optical mirage which can be related to the emergence of an optical vortex in the backscattering direction.
\end{abstract}

DOI: 10.1103/PhysRevA.99.013852

\section{INTRODUCTION}

It is customary to separate the angular momentum (AM) of light [1] into two contributions, the spin angular momentum (SAM) and the orbital angular momentum (OAM), that can be coupled by light propagation and scattering. The study of this spin-orbit interaction (SOI) has attracted a great deal of interest in recent years [2-5].

An interesting analogy between the SOI in light and the spin Hall effect (SHE) in electronic systems can be drawn $[6,7]$. In the latter, electrons with different spins are deflected differently by scattering off impurities due to the SOI. This leads to a transversal spin current that in turn induces a measurable spin accumulation at the sample edges. One of the microscopic origins of the SHE is the so-called side-jump mechanism [8], in which a spin-dependent displacement of the center of mass of the electronic wave packet takes place due to the SOI (for more details, we refer to the reviews $[9,10]$ ).

Similarly, an apparent transversal displacement of a target particle induced by light scattering can be explained by an AM exchange. Hereafter, this effect is referred to as an optical mirage. It has been observed in several situations, for example in beams impinging on a dielectric surface [11-13] or when considering a spherical target described by a single electric polarizability $[14,15]$. In the latter case, the apparent shift of the dipole localization does not depend on the optical properties but rather on the scattering angle, with opposite displacements for incident left and right circularly polarized photons (spins). The apparent shift $(\Delta)$ is maximized at the plane perpendicular to the direction of the incoming wave taking a value of $\Delta=\lambda / \pi$ [15], and thus, for circularly polarized light $[16,17]$, it is always subwavelength. In contrast, for larger multipolar spheres, and certain combinations of radius and refractive index, resonant apparent shifts, reaching tens of wavelengths in magnitude, were found at some specific

\footnotetext{
*juanjo.saenz@dipc.org
}

angles [18]. These results were interpreted [18] as a result of full transfer from SAM to OAM at those directions at which the scattered light is linearly polarized (where the SAM of scattered photons is identically zero).

In this paper, we demonstrate that by taking into account both the electric and magnetic dipoles sustained by a high refractive index (HRI) spherical particle, the subwavelength maximum limit can be drastically surpassed when the particle is excited by circularly polarized light. In other words, a large macroscopic apparent shift $(\Delta \gg \lambda)$, even larger than those reported previously [18], is induced in the backscattering region. Specifically, we show that this optical mirage is related to the generation of a spiraling power flow and can be explained in terms of an angular momentum redistribution per photon between the SAM and OAM contributions.

In contrast with earlier work [18], we show that, for HRI dipolar spheres, the optical mirage is maximum at angles where the spin of the photons is sign-reversed, i.e. at directions at which the light is not linearly polarized.

Based on helicity conservation, we predict an intriguing enhancement of the momentum transfer when the system is dual, i.e., when the electric and magnetic dipolar moments are equal. At this so-called "first Kerker condition" [19-21], the scattered light is circularly polarized in all directions with a vanishing intensity in the backscattering direction. As we show, this leads to a huge apparent shift near backscattering associated with the appearance of a ( $2 \sigma$ charge) topological optical vortex.

\section{SYSTEM AND THEORETICAL METHODS}

We consider a nonabsorbing dielectric sphere of radius $a$ and refractive index $n_{p}$ embedded in an otherwise homogeneous medium with constant and real refractive index $n_{h}$. The geometry of the scattering problem is sketched in Fig. 1, where we consider a circularly polarized plane wave with wave number $k=n_{h} k_{0}=n_{h} 2 \pi / \lambda_{0}$ (where $\lambda_{0}$ is the light wavelength in vacuum) and helicity $\sigma= \pm 1$ (we associate 


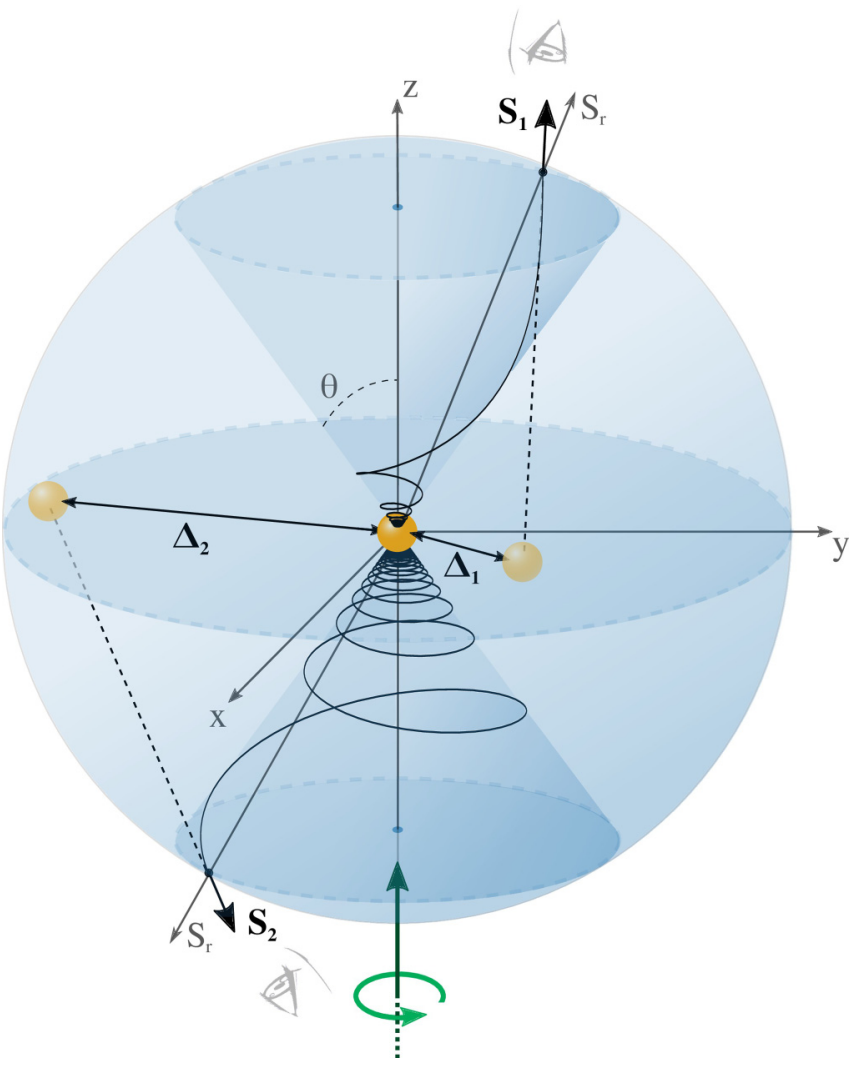

FIG. 1. Schematic representation of the optical mirage vector when considering a clockwise circularly polarized incoming wave (thick green straight arrow lying on the $z$-axis). The observer, represented by an eye, perceives a nonradial scattered Poynting vector $\left(\mathbf{S}_{1}, \mathbf{S}_{2}\right)$ that leads to an apparent shift $\left(\boldsymbol{\Delta}_{1}, \boldsymbol{\Delta}_{2}\right)$ of the dipole localization, both lying on the $x y$ plane.

left polarized light with a positive helicity $\sigma=+1$ ) incident along the $z$-axis. Instead of using the traditional multipole Mie expansion to describe the light scattered by the sphere $[22,23]$, we shall find it useful to work in a basis of multipoles, eigenfunctions, $\boldsymbol{\Psi}_{l m}^{\sigma}$, of the helicity operator $\Lambda[24,25]$,

$$
\boldsymbol{\Lambda} \boldsymbol{\Psi}_{l m}^{\sigma}=(1 / k) \nabla \times \boldsymbol{\Psi}_{l m}^{\sigma}=\sigma \boldsymbol{\Psi}_{l m}^{\sigma},
$$

with

$$
\begin{gathered}
\boldsymbol{\Psi}_{l m}^{\sigma}=\frac{1}{\sqrt{2}}\left[\frac{\nabla \times g_{l}(k r) \mathbf{X}_{l m}}{k}+\sigma g_{l}(k r) \mathbf{X}_{l m}\right] \\
g_{l}(k r)=A_{l}^{(1)} h_{l}^{(1)}(k r)+A_{l}^{(2)} h_{l}^{(2)}(k r), \\
\mathbf{X}_{l m}=\frac{1}{\sqrt{l(l+1)}} \mathbf{L} Y_{l}^{m}(\theta, \varphi),
\end{gathered}
$$

where, following Jackson's notation [22], $\mathbf{X}_{l m}$ denote the vector spherical harmonic, where $\mathbf{X}_{00}=0, g_{l}(k r)$ is a linear combination of the spherical Hankel functions, $Y_{l}^{m}(\theta, \varphi)$ are the spherical harmonics, and $\mathbf{L}$ is the orbital angular momentum operator, $\mathbf{L}=-i(\mathbf{r} \times \nabla)$. In this helicity basis, the incident field can be written as

$$
\frac{\mathbf{E}_{\sigma}^{(0)}}{E_{0}}=\frac{\hat{\boldsymbol{x}}+\sigma i \hat{\boldsymbol{y}}}{\sqrt{2}} e^{i k z}=\sum_{l=0}^{\infty} \sum_{m=-l}^{+l} \sum_{\sigma^{\prime}= \pm 1} C_{l m}^{\sigma \sigma^{\prime}} \boldsymbol{\Psi}_{l m}^{\sigma^{\prime}}
$$

$$
\begin{gathered}
k Z \mathbf{H}_{\sigma}^{(0)}=-i \nabla \times \mathbf{E}_{\sigma}^{(0)}, \\
C_{l m}^{\sigma \sigma^{\prime}}=\sigma i^{l} \sqrt{4 \pi(2 l+1)} \delta_{m \sigma} \delta_{\sigma \sigma^{\prime}},
\end{gathered}
$$

where $1 / Z=\epsilon_{0} c n_{h}$ (where $\epsilon_{0}$ and $c$ are the vacuum permittivity and the speed of light, respectively) and $\boldsymbol{\Psi}_{l m}^{\sigma^{\prime}}$ is given by Eq. (1) with $g_{l}(k r)=j_{l}(k r)$. Such a circularly polarized wave, with helicity $\sigma$, carries a $j_{z}=m=\sigma$ unit of total angular momentum per photon parallel to the propagation direction [22].

In the same basis, the scattered fields are given by

$$
\begin{gathered}
\frac{\mathbf{E}_{\sigma}^{\mathrm{scat}}}{E_{0}}=\sum_{l=0}^{\infty} \sum_{m=-l}^{+l} \sum_{\sigma^{\prime}= \pm 1} D_{l m}^{\sigma \sigma^{\prime}} \boldsymbol{\Psi}_{l m}^{\sigma^{\prime}}, \\
D_{l m}^{\sigma \sigma^{\prime}}=-i^{l} \sqrt{4 \pi(2 l+1)} \frac{\sigma a_{l}+\sigma^{\prime} b_{l}}{2} \delta_{m \sigma},
\end{gathered}
$$

where now, since they are outgoing waves at infinity, $g_{l}(k r)=$ $h_{l}^{(1)}(k r)$. Notice that $a_{l}$ and $b_{l}$ are the standard Mie electric and magnetic scattering coefficients [23]. Since a sphere presents axial symmetry around the $z$-axis, the $j_{z}$ of the incident beam is preserved and the scattered wave can only involve $m=\sigma$. Consequently, $\mathbf{E}_{\sigma}^{\text {scat }}$ is an eigenfunction of the $z$-component of the total (dimensionless) angular momentum operator, $\mathbf{J}=$ $\mathbf{L}+\mathbf{S}^{\text {spin }}$ (as well as of $\mathbf{J}^{2}$ ) [26], with eigenvalue $j_{z}=m=\sigma$,

$$
\begin{gathered}
\sigma=\frac{\mathbf{E}_{\sigma}^{\text {scat* }} \cdot\left(L_{z}+\mathbf{S}_{z}^{\text {spin }}\right) \mathbf{E}_{\sigma}^{\text {scat }}}{\left|\mathbf{E}_{\sigma}^{\text {scat }}\right|^{2}}=\ell_{z}(\mathbf{r})+s_{z}(\mathbf{r}), \\
s_{z}(\mathbf{r})=\frac{-i\left\{\mathbf{E}_{\sigma}^{\text {scat* }} \times \mathbf{E}_{\sigma}^{\text {scat }}\right\} \cdot \hat{\boldsymbol{e}}_{z}}{\left|\mathbf{E}_{\sigma}^{\text {scat }}\right|^{2}}, \\
\ell_{z}(\mathbf{r})=\frac{\mathbf{E}_{\sigma}^{\text {scat* }} \cdot L_{z} \mathbf{E}_{\sigma}^{\text {scat }}}{\left|\mathbf{E}_{\sigma}^{\text {scat }}\right|^{2}}=\frac{-i}{\left|\mathbf{E}_{\sigma}^{\text {scat }}\right|^{2}}\left\{\mathbf{E}_{\sigma}^{\text {scat* }} \cdot \frac{\partial \mathbf{E}_{\sigma}^{\text {scat }}}{\partial \varphi}\right\} .
\end{gathered}
$$

Equation (9) shows that the sum of the (dimensionless) OAM, $\ell_{z}(\mathbf{r})$, and SAM, $s_{z}(\mathbf{r})$, per photon is constant and equal to the helicity of the incoming plane wave. Notice that this is valid even in the near-field region, and it would be valid even in the presence of absorption. However, in general, the helicity is not preserved in the scattering process.

\section{RESULTS AND DISCUSSION}

Let us now consider the scattering from a HRI subwavelength sphere in a spectral range such that the optical response can be described by its first dipolar Mie coefficients $a_{1}$ and $b_{1}$, i.e., by its electric and magnetic polarizabilities $\alpha_{\mathrm{E}}=$ $i a_{1}\left(6 \pi / k^{3}\right)$ and $\alpha_{\mathrm{M}}=i b_{1}\left(6 \pi / k^{3}\right)$. The scattered field can be written as the sum of two components with opposite helicity,

$$
\begin{aligned}
\frac{\mathbf{E}_{\sigma}^{\text {scat }}}{E_{0}} & =-\frac{k^{3}}{\sqrt{12 \pi}}\left\{\left(\sigma \alpha_{\mathrm{E}}+\alpha_{\mathrm{M}}\right) \boldsymbol{\Psi}_{1 \sigma}^{+}+\left(\sigma \alpha_{\mathrm{E}}-\alpha_{\mathrm{M}}\right) \boldsymbol{\Psi}_{1 \sigma}^{-}\right\} \\
& =\mathbf{E}_{\sigma+}+\mathbf{E}_{\sigma-},
\end{aligned}
$$

which in the far-field limit become

$$
\mathbf{E}_{\sigma \sigma^{\prime}} \sim E_{\sigma \sigma^{\prime}} e^{i \sigma \varphi}\left(\hat{\boldsymbol{e}}_{\sigma^{\prime}}+i \sigma \frac{\sqrt{2}}{k r} \frac{\sigma \cos \theta-\sigma^{\prime}}{\sin \theta} \hat{\boldsymbol{e}}_{r}+\cdots\right)
$$


where the last identity corresponds to the medium-far-field expansion with

$$
\begin{gathered}
\frac{E_{\sigma \sigma^{\prime}}}{E_{0}}=\frac{e^{i k r}}{4 \pi k r} k^{3}\left(\frac{\sigma \alpha_{\mathrm{E}}+\sigma^{\prime} \alpha_{\mathrm{M}}}{2}\right)\left(\sigma \cos \theta+\sigma^{\prime}\right), \\
\hat{\boldsymbol{e}}_{\sigma^{\prime}}=\frac{1}{\sqrt{2}}\left(\hat{\boldsymbol{e}}_{\theta}+i \sigma^{\prime} \hat{\boldsymbol{e}}_{\varphi}\right) .
\end{gathered}
$$

The scattered fields by HRI dielectric nanoparticles present a number of peculiar properties arising from the interference between the electric and magnetic dipolar radiation, and they have been largely discussed both theoretically and experimentally [27-33]. Most of these properties are encoded in the far-field radiation pattern, i.e., in the differential scattering cross section given by [20]

$$
\begin{aligned}
\frac{d \sigma_{\text {scat }}(\theta)}{d \Omega} & =\lim _{r \rightarrow \infty} r^{2} \frac{\mathbf{S}^{\text {scat }} \cdot \hat{\boldsymbol{e}}_{r}}{\left|\mathbf{S}^{(0)}\right|}=r^{2} \frac{\left|E_{\sigma+}\right|^{2}+\left|E_{\sigma-}\right|^{2}}{\left|E_{0}\right|^{2}} \\
& =\frac{k^{4}\left|\alpha_{\text {sum }}\right|^{2}}{(4 \pi)^{2}}\left(\frac{1+\cos ^{2} \theta}{2}+2 g \cos \theta\right),
\end{aligned}
$$

where $\mathbf{S}^{\text {scat }}=(1 / 2) \operatorname{Re}\left\{\mathbf{E}^{\text {scat } *} \times \mathbf{H}^{\text {scat }}\right\}$ is the time-averaged Poynting vector, $\left|\alpha_{\text {sum }}\right|^{2} \equiv\left|\alpha_{\mathrm{E}}\right|^{2}+\left|\alpha_{\mathrm{M}}\right|^{2}$, and

$$
g=\frac{\operatorname{Re}\left\{\alpha_{\mathrm{E}} \alpha_{\mathrm{M}}^{*}\right\}}{\left|\alpha_{\text {sum }}\right|^{2}}
$$

is the so-called asymmetry factor [23] for dipolar electric and magnetic scatterers [20,34].

Although in the strict far-field limit the flow lines of $\mathbf{S}^{\text {scat }}$ lie along the spherical radial direction, tracing them to their source they do indeed spiral toward the origin in analogy with the light scattered by an electric dipole excited by circularly polarized light $[15,18,35-37]$. Consequently, as sketched in Fig. 1, the full Poynting vector $\mathbf{S}^{\text {scat }}$ makes an angle with the line of sight, which determines an apparent shift $\Delta$ in the perceived position of the particle, with

$$
\begin{aligned}
\boldsymbol{\Delta} & =\lim _{k r \rightarrow \infty}-r\left(\frac{\mathbf{S}^{\text {scat }}-\hat{\boldsymbol{e}}_{r}\left(\hat{\boldsymbol{e}}_{r} \cdot \mathbf{S}^{\text {scat }}\right)}{\left|\mathbf{S}_{r}\right|}\right) \\
& =\lim _{k r \rightarrow \infty}\left(\frac{\hat{\boldsymbol{e}}_{r} \times\left(\mathbf{r} \times \mathbf{S}^{\text {scat }}\right)}{\left|\mathbf{S}_{r}\right|}\right) \\
& =\lim _{k r \rightarrow \infty}\left(\frac{2 i}{k\left|\mathbf{E}_{\sigma}^{\text {scat }}\right|^{2}} \frac{\mathbf{E}_{\sigma}^{\text {scat }} *}{\sin \theta} \cdot \frac{\partial \mathbf{E}_{\sigma}^{\text {scat }}}{\partial \varphi}\right) \hat{\boldsymbol{e}}_{\varphi},
\end{aligned}
$$

where $\mathbf{E}_{\sigma}^{\text {scat }}$ is given by Eqs. (12) and (13). Taking into account Eq. (11), the apparent shift can be written as

$$
\begin{aligned}
\frac{\Delta}{(\lambda / \pi)} & =-\frac{\ell_{z}(\theta)}{\sin \theta} \hat{\boldsymbol{e}}_{\varphi}=\frac{s_{z}(\theta)-\sigma}{\sin \theta} \hat{\boldsymbol{e}}_{\varphi} \\
& =-\sigma\left[\frac{\sin \theta(1+2 g \cos \theta)}{1+\cos ^{2} \theta+4 g \cos \theta}\right] \hat{\boldsymbol{e}}_{\varphi} .
\end{aligned}
$$

This is the first important result of this paper: the shift is always along $\hat{\boldsymbol{e}}_{\varphi}$, perpendicular to the incidence plane and proportional to the $z$-component of the OAM per photon. Importantly, the sign of the displacement is purely determined by the incoming helicity.

In the absence of magnetic dipolar response, setting $g=0$ in Eq. (22), one recovers the previously reported results for electric dipoles [15,18], which were interpreted as a result of transfer from SAM to OAM $[18,38]$. According to those

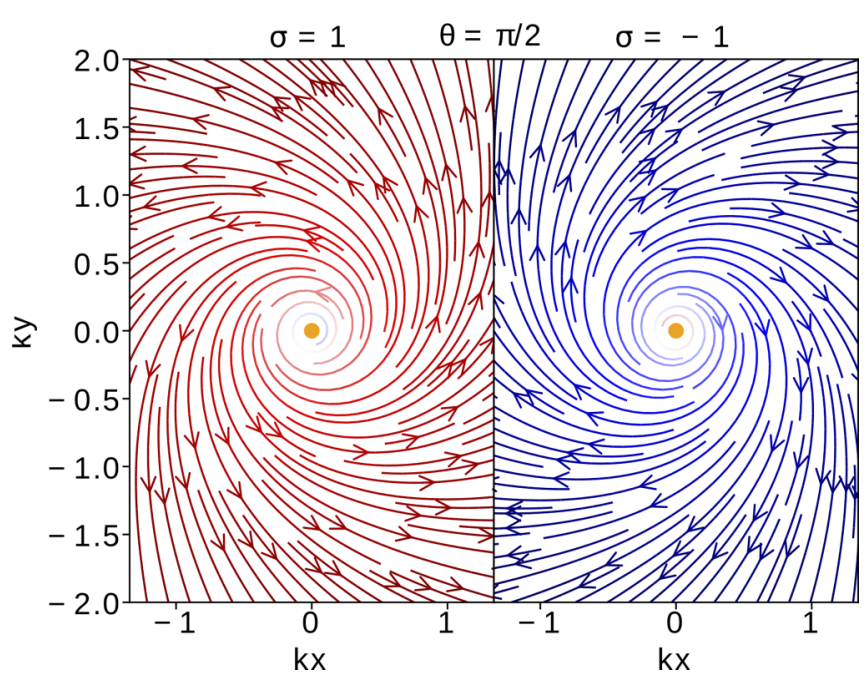

FIG. 2. Poynting vector streamlines with counterclockwise (clockwise) rotation for $\sigma=1(\sigma=-1)$ when viewed from the perpendicular direction, $\theta=\pi / 2$. The streamlines lie on the $x-y$ plane ( $k x$ and $k y$ are dimensionless variables, where $k=2 \pi / \lambda$ is the light wave number). This figure is valid for any dipolar response, i.e., arbitrary $\alpha_{\mathrm{E}}$ and $\alpha_{\mathrm{M}}$. The small central circle represents the dipolar particle.

previous works, this transfer is expected to be maximum at those directions at which the scattered light is linearly polarized (where the SAM of scattered photons is identically zero). For an electric dipole excited by circularly polarized light, the maximum transfer would take place in the plane perpendicular to the incoming light $(\theta=\pi / 2)$, where the maximum displacement is equal to $\Delta=\lambda / \pi$.

The fields scattered by electric and magnetic dipoles present a very different polarization structure $[39,40]$. Contrary to the purely electric (or magnetic) case, when excited with a circularly polarized field, the scattered radiation on the plane perpendicular to the incoming light $(\theta=\pi / 2)$ is no longer linearly polarized. Interestingly, this change does not affect the streamlines of the Poynting vector on this particular plane (as shown in Fig. 2), leading to the same subwavelength optical mirage. However, out of this plane the apparent displacement presents a peculiar behavior that depends strongly on both $\theta$ and the wavelength

Figures 3 and 4 summarize the anomalous behavior of the apparent displacement $\Delta(\lambda, \theta)$ for silicon nanospheres in the infrared (similar behavior is obtained in other spectral ranges as long as the scattering cross section can be described by only the first two dipolar multipoles [see Fig. 3(a)]). As can be seen in Fig. 4, for $\theta=\pi / 2$ the displacement is always $\lambda / \pi$ for all wavelengths. When the asymmetry factor $g$ is negative $\left(\lambda_{g 1}<\lambda<\lambda_{g 2}\right)$, the maximum displacement occurs for $\theta<\pi / 2$ and it is always subwavelength but slightly larger than the one for $\theta=\pi / 2$. However, for $g>0$ the apparent displacement can be much larger than $\lambda / \pi$, and when the electric and magnetic polarizabilities are identical $\left(\lambda=\lambda_{\mathrm{K} 1}\right)$, i.e., at the so-called first Kerker condition, it diverges as $\theta \rightarrow \pi$. Notice that the singularity is resolved naturally since at the first Kerker condition there is exactly zero backscattered intensity. 


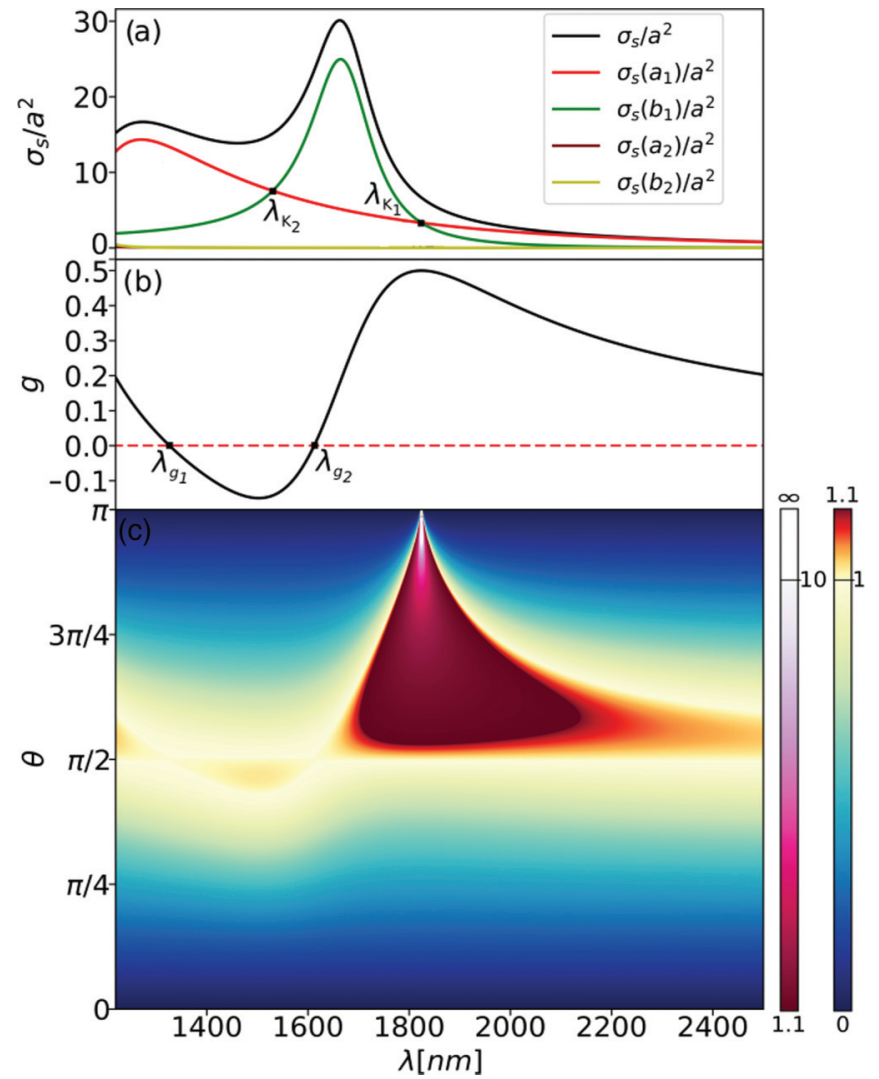

FIG. 3. (a) Scattering cross sections $\sigma_{\mathrm{s}}$ for a $230 \mathrm{~nm} \mathrm{Si}$ nanosphere vs the wavelength. The special wavelengths $\lambda_{\mathrm{K}_{1}}=$ $1825 \mathrm{~nm}$ and $\lambda_{\mathrm{K}_{2}}=1520 \mathrm{~nm}$ correspond to first and second Kerker conditions, respectively. (b) Asymmetry factor vs the wavelength. This is identical to zero at $\lambda_{\mathrm{g}_{1}}=1326 \mathrm{~nm}$ and $\lambda_{\mathrm{g}_{2}}=1612 \mathrm{~nm}$ (and negative in between). The maximum value is localized at the first Kerker condition, namely $\lambda_{\mathrm{K}_{1}}$. (c) Colormap of the normalized optical mirage, $\Delta /(\lambda / \pi)$, vs the scattering angle and the wavelength. The maximum enhancement for $\lambda_{\mathrm{K}_{1}}$ at backscattering $(\theta=\pi)$ is clearly observed.

We can now examine the peculiar behavior of $\Delta$ near the first Kerker condition in terms of the angular momentum flow. When the electric and magnetic responses are identical, i.e., $\alpha_{\mathrm{E}}=\alpha_{\mathrm{M}}$, the system is "dual" and the scattering preserves helicity $[41,42]$. In this case, the asymmetry factor is maximum, $g=1 / 2$ [see Fig. 3(b)], which leads to $s_{z}(\theta)=\sigma \cos \theta$ and

$$
\frac{\Delta \pi}{\lambda_{\mathrm{K}_{1}}}=-\sigma \tan \left(\frac{\theta}{2}\right) \hat{\boldsymbol{e}}_{\varphi} .
$$

From this equation, two interesting limiting cases can be identified: First, in the forward direction the optical mirage and $l_{\mathrm{z}}$ go to zero since $\mathbf{S}_{\varphi}=\mathbf{0}$. This can alternatively be understood by means of the symmetries of the system: due to the duality of the scatterer, the system must conserve the helicity of the incoming field, which in the forward direction corresponds to the spin density. Thus, the incident circular polarization is preserved in the forward direction and must carry all the angular momentum density (leaving $\ell_{\mathrm{z}}=0$ ). Second, in the direction perpendicular to the incident wave vector $(\theta=\pi / 2)$, the interference term vanishes. As a consequence, $s_{z}=0$ and $\ell_{z}=\sigma$, and, in analogy with electric

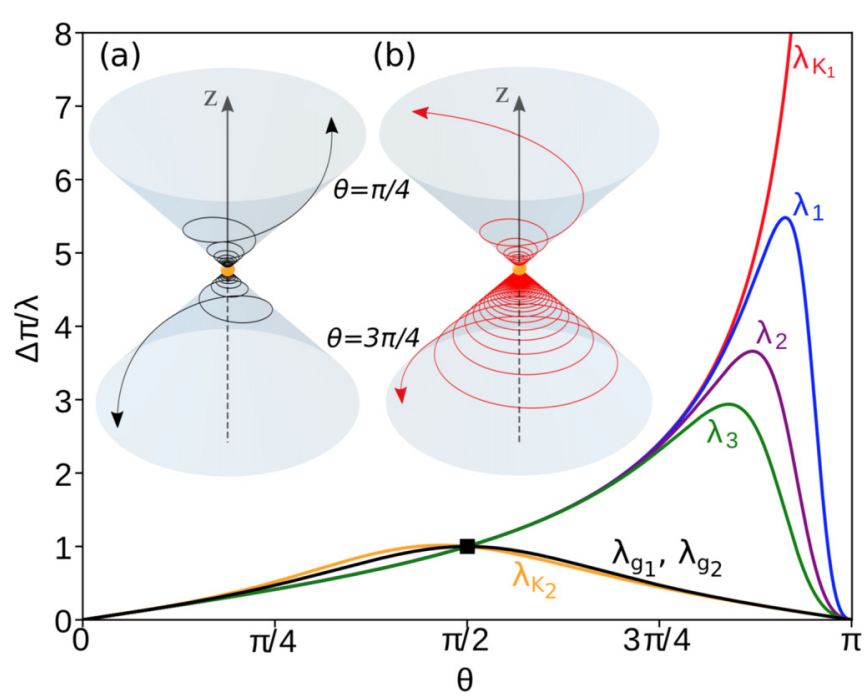

FIG. 4. Optical mirage colormap (Fig. 3) cuts vs the scattering angle for different values of the wavelength, belonging to regions with $g<0\left(\lambda_{\mathrm{K}_{2}}\right), g=0\left(\lambda_{\mathrm{g}_{1}}, \lambda_{\mathrm{g}_{2}}\right)$, and $g>0\left(\lambda_{1}, \lambda_{2}\right.$, and $\lambda_{3}$, respectively decreased 5,10 , and $15 \mathrm{~nm}$ with respect to $\lambda_{\mathrm{K}_{1}}$, and $\lambda_{\mathrm{K}_{1}}$ itself). At $\theta=\pi / 2, \Delta=\lambda / \pi$ is observed to be a universal value. Both subplots show examples of trajectories of the Poynting vector at forward and backscattering, being similar for $\lambda_{\mathrm{g}_{1}}$ and $\lambda_{\mathrm{g}_{2}}$ (a) and considerably different for $\lambda_{\mathrm{K}_{1}}$ (b).

dipoles, we obtain $\Delta=\sigma \lambda / \pi$, although in that case light in this direction is fully circularly polarized (see Fig. 2).

The most striking effect arises at an observation angle near backscattering $\theta \lesssim \pi$ where, as discussed above, the apparent displacement diverges. This divergence is solved because the Poynting vector becomes strictly zero at backscattering, which suggests the appearance of an optical vortex in that direction. As a matter of fact, near backscattering $\ell_{z}(\lesssim \theta) \rightarrow$ $2 \sigma$, while the spin reverses sign $s_{z}(\theta \lesssim \pi) \rightarrow-\sigma$ (but still maintaining constant helicity), which confirms the existence of a vortex with $l=2 \sigma$ emerging from a nanoparticle as a nanoscale analog of the light backscattered from a perfect reflecting cone [43].

\section{CONCLUDING REMARKS}

In conclusion, we have shown that light scattering from dipolar electric and magnetic nanoparticles, excited by circular polarized light, can lead to macroscopic apparent displacements of the particle position ("optical mirages") much larger than the incident wavelength. We derived an explicit relationship between the apparent shift and the $z$-component of the OAM per photon, $\ell_{z}$. As a result of the interference between the fields scattered by the electric and magnetic dipoles, we found that $\ell_{z}$ presents a nontrivial dependence on the scattering angle, which, in contrast with previous work [18], leads to optical mirage maxima at angles where the spin of the photons is sign-reversed, i.e., at directions at which the light is not linearly polarized. Interestingly, for dual spheres, i.e., at the so-called first Kerker condition, we predict a huge enhancement of the apparent shift related to the emergence of an optical vortex in the backscattering direction. We believe that our results open new perspectives in the study of optical 
spin-orbit phenomena, including new possible applications of HRI particles as building blocks in photonic devices.

\section{ACKNOWLEDGMENTS}

This research was supported by the Spanish Ministerio de Economía y Competitividad (MICINN) and European Regional Development Fund (ERDF) Projects
No. FIS2014-55987-P, No. FIS2015-69295-C3-3-P, and No. FIS2017-82804-P, by the Basque Dep. de Educación Project No. PI-2016-1-0041 and by the Basque Government ELKARTEK program (KK-2016/00030, KK-2017/00089). A.G.-E. received funding from the Fellows Gipuzkoa fellowship of the Gipuzkoako Foru Aldundia through FEDER "Una Manera de hacer Europa."

J.O.-T. and C.S.-F. contributed equally to this work.
[1] L. Allen, S. M. Barnett, and M. J. Padgett, Optical Angular Momentum (CRC, Boca Raton, FL, 2003).

[2] V. S. Liberman and B. Y. Zel'dovich, Phys. Rev. A 46, 5199 (1992).

[3] J. H. Crichton and P. L. Marston, Electron. J. Diff. Eqs. Conf. 04, 37 (2000).

[4] M. Berry, M. Jeffrey, and M. Mansuripur, J. Opt. A 7, 685 (2005).

[5] K. Y. Bliokh, F. Rodríguez-Fortuño, F. Nori, and A. V. Zayats, Nat. Photon. 9, 796 (2015).

[6] M. Dyakonov and V. Perel, Phys. Lett. A 35, 459 (1971).

[7] J. E. Hirsch, Phys. Rev. Lett. 83, 1834 (1999).

[8] L. Berger, Phys. Rev. B 2, 4559 (1970).

[9] M. I. Dyakonov and A. Khaetskii, in Spin Physics in Semiconductors, edited by M. I. Dyakonov (Springer-Verlag, Berlin, Heidelberg, 2008), pp. 211-243.

[10] J. Sinova, S. O. Valenzuela, J. Wunderlich, C. Back, and T. Jungwirth, Rev. Mod. Phys. 87, 1213 (2015).

[11] M. Onoda, S. Murakami, and N. Nagaosa, Phys. Rev. Lett. 93, 083901 (2004).

[12] K. Y. Bliokh and Y. P. Bliokh, Phys. Rev. Lett. 96, 073903 (2006).

[13] O. Hosten and P. Kwiat, Science 319, 787 (2008).

[14] C. Schwartz and A. Dogariu, Opt. Express 14, 8425 (2006).

[15] H. F. Arnoldus, X. Li, and J. Shu, Opt. Lett. 33, 1446 (2008).

[16] In contrast, the apparent shifts for electric dipolar particles illuminated by "elliptically" polarized fields can be much larger, as recently reported by Araneda et al. [17] after the submission of the present work.

[17] G. Araneda, S. Walser, Y. Colombe, D. Higginbottom, J. Volz, R. Blatt, and A. Rauschenbeutel, Nat. Phys. 15, 17 (2019).

[18] D. Haefner, S. Sukhov, and A. Dogariu, Phys. Rev. Lett. 102, 123903 (2009).

[19] M. Kerker, D.-S. Wang, and C. Giles, J. Opt. Soc. Am. A 73, 765 (1983).

[20] M. Nieto-Vesperinas, R. Gomez-Medina, and J. J. Saenz, J. Opt. Soc. Am. A 28, 54 (2011).

[21] R. Gomez-Medina, B. Garcia-Camara, I. Suárez-Lacalle, F. González, F. Moreno, M. Nieto-Vesperinas, and J. J. Sáenz, J. Nanophoton. 5, 053512 (2011).

[22] J. D. Jackson, Classical Electrodynamics (Wiley, New York, 1999).

[23] C. F. Bohren and D. R. Huffman, Absorption and Scattering of Light by Small Particles (Wiley, New York, 2008).
[24] I. Fernandez-Corbaton, X. Zambrana-Puyalto, and G. Molina-Terriza, Phys. Rev. A 86, 042103 (2012).

[25] X. Zambrana-Puyalto, I. Fernandez-Corbaton, M. Juan, X. Vidal, and G. Molina-Terriza, Opt. Lett. 38, 1857 (2013).

[26] N. Tischler, X. Zambrana-Puyalto, and G. Molina-Terriza, Eur. J. Phys. 33, 1099 (2012).

[27] A. B. Evlyukhin, C. Reinhardt, A. Seidel, B. S. Luk'yanchuk, and B. N. Chichkov, Phys. Rev. B 82, 045404 (2010).

[28] A. García-Etxarri, R. Gómez-Medina, L. S. Froufe-Pérez, C. López, L. Chantada, F. Scheffold, J. Aizpurua, M. Nieto-Vesperinas, and J. J. Sáenz, Opt. Express 19, 4815 (2011).

[29] J.-M. Geffrin, B. García-Cámara, R. Gómez-Medina, P. Albella, L. S. Froufe-Pérez, C. Eyraud, A. Litman, R. Vaillon, F. González, M. Nieto-Vesperinas, J. J. Sáenz, and F. Moreno, Nat. Commun. 3, 1171 (2012).

[30] S. Person, M. Jain, Z. Lapin, J. J. Sáenz, G. Wicks, and L. Novotny, Nano Lett. 13, 1806 (2013).

[31] Y. H. Fu, A. I. Kuznetsov, A. E. Miroshnichenko, Y. F. Yu, and B. Luk'yanchuk, Nat. Commun. 4, 1527 (2013).

[32] L. Shi, J. T. Harris, R. Fenollosa, I. Rodriguez, X. Lu, B. A. Korgel, and F. Meseguer, Nat. Commun. 4, 1904 (2013).

[33] A. I. Kuznetsov, A. E. Miroshnichenko, M. L. Brongersma, Y. S. Kivshar, and B. Luk'yanchuk, Science 354, aag2472 (2016).

[34] R. Gómez-Medina, L. S. Froufe-Pérez, M. Yépez, F. Scheffold, M. Nieto-Vesperinas, and J. J. Sáenz, Phys. Rev. A 85, 035802 (2012).

[35] W. Gough, Eur. J. Phys. 7, 81 (1986).

[36] H. F. Arnoldus and J. T. Foley, Opt. Commun. 231, 115 (2004).

[37] C. Schwartz and A. Dogariu, Opt. Lett. 31, 1121 (2006).

[38] K. Y. Bliokh, E. A. Ostrovskaya, M. A. Alonso, O. G. Rodríguez-Herrera, D. Lara, and C. Dainty, Opt. Express 19, 26132 (2011).

[39] A. Garcia-Etxarri, ACS Photon. 4, 1159 (2017).

[40] A. García-Etxarri and J. A. Dionne, Phys. Rev. B 87, 235409 (2013).

[41] I. Fernandez-Corbaton, X. Zambrana-Puyalto, N. Tischler, X. Vidal, M. L. Juan, and G. Molina-Terriza, Phys. Rev. Lett. 111, 060401 (2013).

[42] M. K. Schmidt, J. Aizpurua, X. Zambrana-Puyalto, X. Vidal, G. Molina-Terriza, and J. J. Sáenz, Phys. Rev. Lett. 114, 113902 (2015).

[43] M. Mansuripur, A. R. Zakharian, and E. M. Wright, Phys. Rev. A 84, 033813 (2011). 